\title{
La priorización de comportamientos para mejorar programas de cambio social y de comportamiento durante una emergencia de salud pública: un llamado a la acción
}

Breakthrough RESEARCH

Follow this and additional works at: https://knowledgecommons.popcouncil.org/departments_sbsr-pgy

Part of the Health Communication Commons, and the Public Health Commons

How does access to this work benefit you? Let us know!

\section{Recommended Citation}

Breakthrough RESEARCH. 2019. "La priorización de comportamientos para mejorar programas de cambio social y de comportamiento durante una emergencia de salud pública: un llamado a la acción," Informe Programático. Washington, DC: Population Council. 


\title{
La priorización de comportamientos para mejorar programas de cambio social y de comportamiento durante una emergencia de salud pública: un Ilamado a la acción
}

\begin{abstract}
A medida que los especialistas en desarrollo internacional continúan trabajando en los desafíos de la salud pública y nuevas enfermedades emergentes, es de suma importancia la priorización de comportamientos. Este breve informe tiene como objetivo argumentar por qué es importante la priorización de comportamientos para fortalecer los programas de cambio social y de comportamiento (CSC). El documento finaliza con un llamado a la acción a que diferentes actores interesados aboguen por y participen en procesos que prioricen comportamientos como parte del diseño, planificación y adaptación de programas de cambio social y de comportamiento.
\end{abstract}

\section{ELEMENTOS CLAVE}

- Un paso esencial que los programas de CSC deben llevar a cabo es identificar y priorizar comportamientos clave, y focalizar audiencias prioritarias, para así aumentar su impacto y alcanzar los resultados de comportamiento y salud deseados.

- Promover demasiados comportamientos puede potencialmente llevar a resultados ineficaces 0 incluso contraproducentes, ya que cuando se les pide a las personas realizar demasiados comportamientos pueden tener menos probabilidades de cambiar cualquiera de ellos.

Durante el brote de Zika en América Latina, una revisión de los programas apoyados por la Agencia para el Desarrollo Internacional de los Estados Unidos (USAID) en el 2017 identificó más de 30 comportamientos y mensajes preventivos contra el Zika que estaban siendo promovidos por socios implementadores en los distintos países. ${ }^{1}$ El mensaje entregado sobre comportamientos deseados frecuentemente era bastante general, como "mantenga limpio su patio", en vez de un mensaje específico, como "elimine las fuentes de agua estancada de su patio". Esto generó confusión en las poblaciones observadas y una falta de consistencia en los mensajes y materiales entregados por los socios de USAID. Estudios muestran que cuando se les pide a las personas realizar demasiados comportamientos, tienen menos probabilidades de cambiar cualquiera de ellos, ${ }^{2}$ ya que enfrentan dificultades para elegir qué comportamiento modificar, un fenómeno conocido en la literatura como conflicto de elección. ${ }^{3}$ Además, cuando se entregan mensajes vagos (como "limpiar su patio") los comportamientos deseados dependen de la interpretación de la persona que recibe el mensaje, quien podría elegir, por ejemplo, barrer o eliminar la basura de su patio en vez de realizar el comportamiento realmente deseado: eliminar agua estancada. Las ciencias del comportamiento, el marketing social, el diseño centrado en las personas y otras especialidades de investigación han demostrado que entregar mensajes claros, eligiendo de una breve lista de comportamientos de manera clara, concisa, específica y consistente tiene un mejor impacto en el comportamiento de las personas. ${ }^{4}$

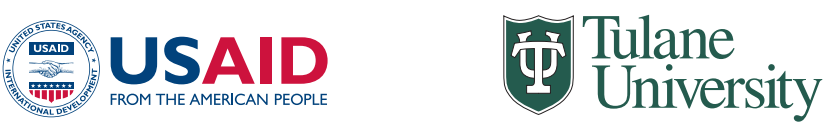

Mediante un proceso consultivo, basado en evidencia empírica, USAID y un equipo de socios implementadores de respuesta al Zika de diversas especialidades técnicas (entomológica, clínica, CSC) priorizaron siete comportamientos de prevención clave dentro de tres grandes categorías (protección personal, control del vector en hogares y comunidades, y comportamientos propicios a la prevención). ${ }^{1}$ Los criterios utilizados para evaluar los comportamientos incluyeron: efectividad del comportamiento en prevenir de la transmisión del arbovirus o en la reducción de la población de vectores; atributos varios (como la frecuencia que un comportamiento requiere ser realizado para ser efectivo); viabilidad del comportamiento (pasos simples o múltiples; interacción con otras personas); y disponibilidad y accesibilidad de los materiales necesarios para el llevar a cabo el comportamiento (ver Cuadro 1). Los siete comportamientos prioritarios, validados entre los socios implementadores, son:

1. Usar repelente de mosquitos.

2. Usar el condón durante el embarazo para prevenir la transmisión sexual.

3. Eliminar regularmente el agua estancada que se acumula dentro y fuera de la casa.

4. Cubrir los recipientes de almacenamiento de agua en todo momento con una tapa ajustada.

5. Eliminar semanalmente los huevos de mosquito de las paredes de los recipientes de almacenamiento de agua.

6. Asistir a las consultas de control prenatal para el seguimiento del embarazo.

7. Buscar consejería sobre los métodos de planificación familiar si no planea quedar embarazada.

Los actores que han participado en la respuesta al Zika concluyeron que el ejercicio de priorizar comportamientos, y el

\section{CUADRO 1 ATRIBUTOS DEL COMPORTAMIENTO}

Los atributos de un comportamiento-tales como su factibilidad, si necesita ser repetido o no, el nivel de habilidad requerido, sus costos de oportunidad y su dependencia de otros-pueden afectar si una persona adoptará un nuevo comportamiento o modificará uno existente.
POPULATION COUNCIL

Ideas. Evidence. Impact.
Breakthrough

RESEARCH FOR SOCIAL \& BEHAVIOR CHANG 
proceso posterior de adaptar los programas de CSC, ha sido extremadamente exitoso para focalizar los esfuerzos.

\section{Llamado a la acción}

Durante una emergencia de salud pública, la priorización de comportamientos basada en evidencia empírica es un paso necesario para lograr los resultados de comportamiento y bienestar deseados. Recomendamos que los actores participantes de las ciencias sociales y clínicas, los socios implementadores de programas, los actores interesados del gobierno y la comunidad en general deben unirse para acordar un enfoque prioritario, basado en evidencia, que de manera práctica agrupe los comportamientos factibles para poblaciones objetivo. La Tabla 1 presenta los actores involucrados y las acciones para abogar y emplear la priorización del comportamiento como un paso clave en programas de cambio social y de comportamiento.

\section{TABLA 1 LLAMADO A LA ACCIÓN}

\begin{tabular}{|c|c|}
\hline DONANTES & $\begin{array}{l}\text { - Incluir y abogar por la priorización de comportamientos como parte integral del diseño de programas } \\
\text { junto a socios implementadores. } \\
\text { - Facilitar un proceso de priorización de comportamientos para asegurar la coordinación y alineamiento } \\
\text { de los programas de CSC entre socios. }\end{array}$ \\
\hline GOBIERNO Y POLÍTICOS & $\begin{array}{l}\text { - Usar la evidencia de la priorización de comportamiento para influir y actualizar las estrategias y políti- } \\
\text { cas clave según corresponda. }\end{array}$ \\
\hline IMPLEMENTADORES DE CSC & $\begin{array}{l}\text { - Abogar por que la priorización de comportamientos sea un elemento central para el diseño y la planifi- } \\
\text { cación de programas de CSC realizados entre múltiples socios. } \\
\text { - Revisar resultados de investigaciones y evidencia empírica como parte del proceso de priorización de } \\
\text { comportamientos. } \\
\text { - Documentar experiencias de implementación y ejemplos exitosos del efecto de la priorización de } \\
\text { comportamientos como una estrategia clave de los programas de CSC. } \\
\text { - Monitorear y evaluar el impacto programático de la priorización de comportamientos. }\end{array}$ \\
\hline $\begin{array}{l}\text { OTROS ACTORES } \\
\text { INVOLUCRADOS EN SALUD } \\
\text { PÚBLICA Y DESARROLLO } \\
\text { INTERNACIONAL }\end{array}$ & $\begin{array}{l}\text { - Participar en procesos de priorización de comportamientos para integrar experiencia técnica donde la } \\
\text { evidencia empírica sea insuficiente. }\end{array}$ \\
\hline $\begin{array}{l}\text { UNIVERSIDADES Y } \\
\text { CENTROS DE INVESTIGACIÓN }\end{array}$ & $\begin{array}{l}\text { - Encontrar, sintetizar y compartir hallazgos empíricos para informar la priorización de } \\
\text { comportamientos. } \\
\text { - Participar en el proceso de priorizar comportamientos para ayudar en la interpretación de datos. } \\
\text { - Evaluar el impacto programático de la priorización de comportamientos. }\end{array}$ \\
\hline
\end{tabular}

\section{References}

${ }^{1}$ Pinchoff, J. et al. 2019. "Evidence-based process for prioritizing behavior-change messages: Zika prevention in Latin America and the Caribbean and applicability to future health emergency responses," Global Health: Science and Practice 7(3):404-417; https://doi.org/10.9745/GHSP-D-19-00188

2McDonald, J. et al. 2017. "Recalling and intending to enact health recommendations: optimal number of prescribed behaviors in multibehavior messages," Clinical Psychological Science 5(5): 858-865.

${ }^{3}$ Tversky, A. and E. Shafir. 1992. "Choice under conflict: the dynamics of deferred decision," Psychological Science 3: 358-361.

${ }^{4}$ Piotrow, P. T. et al. 1997. Health Communication: lessons from family planning and reproductive health. Westport, CT: Praeger.

${ }^{5}$ Silva, M. et al. 2019. "Lessons learned from the SBC programming in the US AID Zika response," Technical Report. Washington DC: Population Council, Breakthrough RESEARCH.

${ }^{6}$ Edmeades, J. \& S. Mathur. 2019. "Insights to improve social and behavior change programming through a focus on the attributes of behaviors," Research Brief. Washington DC: Population Council, Breakthrough RESEARCH.
Breakthrough RESEARCH

Nuestro proyecto convierte la evidencia en acción al guiar programas y políticas de cambio social y de comportamiento, con el objetivo de mejorar el costo-efectividad de las estrategias de salud y desarrollo de la Agencia para el Desarrollo Internacional de los Estados Unidos (USAID). El proyecto de investigación Breakthrough RE. SEARCH cataliza los programas de cambio social y de comportamiento mediante investigaciones y evaluaciones de punta y promoviendo soluciones basadas en evidencia empírica para mejorar la salud y el desarrollo internacional.

Breakthrough RESEARCH es posible gracias al generoso apoyo del pueblo estadounidense a través de la Agencia de los Estados Unidos para el Desarrollo Internacional (USAID), bajo los términos del acuerdo de cooperación no. AID-0AA-A-17-00018.

El contenido de este documento es responsabilidad exclusiva de Breakthrough RESEARCH y el Population Council y no refleja necesariamente los puntos de vista de la Agencia para el Desarrollo Internacional de los Estados Unidos (USAID) o del Gobierno de los Estados Unidos.

Population Council

4301 Connecticut Avenue, NW | Suite 280 | Washington, DC 20008

Tel: +1 2022379400 | breakthroughresearch@popcouncil.org

breakthroughactionandresearch.org 